\title{
Sans objet d'Aurélien Bory : pour une chorégraphie intermédiale
}

Oriane MAUBERT, Université Montpellier 3 Paul-Valéry, Laboratoire RIRRA 21

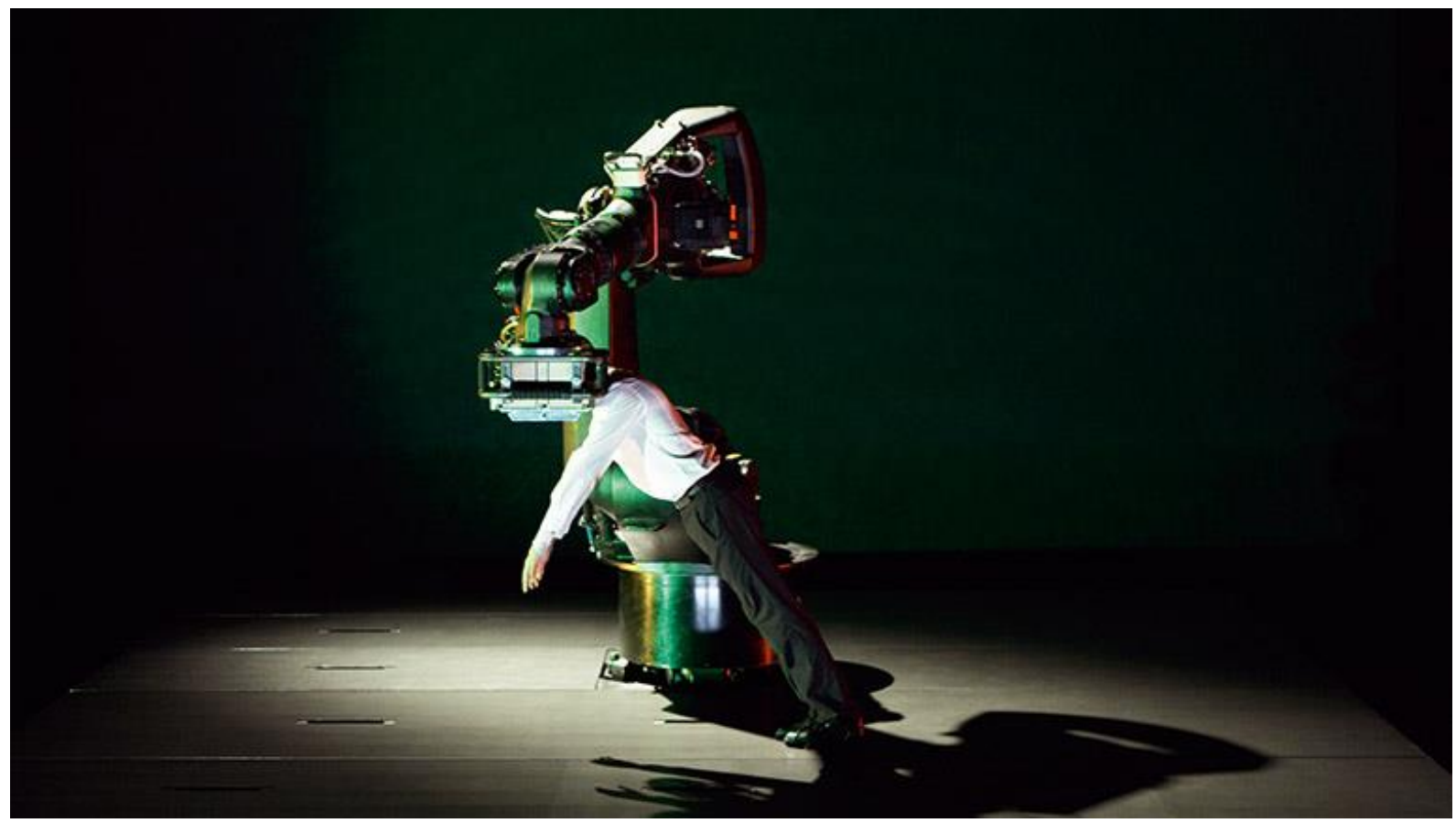

Crédits : Aglaé Bory (C)

Notre désir est de confronter le spectateur à une naissance quasi divine du mouvement dans un corps connecté au cosmos et, surtout, d'ouvrir par là un questionnement philosophique. (Plassard 65)

De la marionnette à la danse sur la scène contemporaine, il n'y a peut-être qu'un pas. L'observation du paysage artistique est révélatrice : créant un nouveau langage corporel autant que sculptural, les spectacles chorégraphico-marionnettiques offrent à voir des corps entre cohabitation, répulsion, fusion, permettant de se pencher sur la manière dont un mouvement chorégraphique commun émerge, comme on peut l'observer dans les créations de Nicole Mossoux, Josef Nadj, Michèle Anne de Mey ou Denis Plassard, parmi d'autres. Par ces zones de rencontres situant les corps et les espaces au seuil, dans une instabilité des danseurs et des formes, chacun semble créer une manière de danser la marionnette, de marionnettiser un corps chorégraphique. L'intime, sensoriel et énigmatique, de la naissance de l'impulsion du mouvement vient alors rencontrer la composition sculpturale de la marionnette (qui peut être gémellaire parfois, fusionnelle souvent). Une respiration commune émerge de cette rencontre, dégourdissant les jambes trop longtemps restées inanimées, les bras tant de fois trop étirés, réveillant des corps plongés jusqu'ici dans les chemins autonomes - presque automates - qui étaient les leurs. Ici, un mouvement commun naît. 
Il s'agit ici de se concentrer sur une partie du travail d'Aurélien Bory, présenté ainsi par le journaliste Nicolas Martin sur France Culture lors d'un entretien avec l'artiste : « [Vous êtes un] metteur en scène, chorégraphe [ayant] fondé la Compagnie 111 à Toulouse en 2000. Vous travaillez depuis le départ sur ce qu'on appelle une hybridation des genres, pour que le spectateur ne puisse pas reconnaître la forme artistique ou la discipline que vous lui proposez. Vous parlez vous-même de théâtre physique ». De formation à l'origine scientifique, Bory s'est notamment intéressé à la physique et à l'acoustique avant de se diriger vers les arts du cirque ${ }^{1}$.

Le spectacle Sans objet créé en 2009 par Bory nous préoccupe ici révélant, en creux ou de manière assumée, un geste de manipulation propre à la marionnette autant qu'un geste chorégraphique. L'œuvre de Bory semble se nourrir d'approches artistiques diverses, approfondies au détour des rencontres d'un parcours artistique touchant autant à la danse qu'aux arts du cirque, à l'espace et au matériau dans leurs rencontres avec le corps, ne déterminant pas alors la définition de son art.

Comme Bory le dit lui-même dans son dossier de presse : «La relation entre l'être humain et la technologie se déploie considérablement. Là où il existait une frontière $[\ldots]$, à savoir celle entre l'inerte et le vivant, on voit apparaître une zone de latence [...]. L'homme, et ses deux réalités, le corps et la pensée, - n'est plus seul»(5). Dans une dynamique chorégraphique similaire à la création Transports exceptionnels, duo pour un danseur et une pelleteuse de Dominique Boivin (2005), Sans objet de Bory propose la rencontre chorégraphique de deux danseurs avec un robot industriel détaché de sa fonction d'origine : le robot, sorti de l'usine de conception de voitures à laquelle il était affecté, se retrouve sans objet, dépossédé de son utilité, dans la nudité et l'isolement extrêmes face au monde.

Néanmoins, la comparaison avec l'œuvre de Boivin s'arrête là : si les deux pièces chorégraphiques jouent de leur aspect spectaculaire au sein duquel le danseur frôle en permanence le danger face à la machine, Boivin cherche à créer une illusion d'animation en dérobant le machiniste à la vue du spectateur derrière des vitres sans teint, là où le manipulateur chez Bory entre, nous le verrons, dans un jeu de caché-montré. L'illusion de l'animation est ici, dès le départ, renégociée, voire réinterrogée chez Bory dans ce jeu de dialogue chorégraphique entre robot et danseur, objet en mouvement et corps vivant, se détournant alors, par le geste chorégraphique, de la question de l'inertie présente en marionnette ${ }^{2}$.

De quelle façon cette rencontre intermédiale entre danseurs et robot parvient-elle à créer une confrontation chorégraphique entre ces deux corps, l'un de chair, l'autre de matière, $a$ priori opposés ? Cette question de l'intermédialité est notamment soulevée par la définition que propose Patrice Pavis (2014), à savoir un «assemblage dynamique de matériaux 
juxtaposés » (152). On peut apporter une précision, relative à Sans objet, en désignant la forme intermédiale comme se situant entre plusieurs médias, à l'interstice de disciplines (ici danse, marionnette et robotique), au seuil de corps humain et non humain, une forme qui n'est plus tout à fait là et déjà dans l'ouverture vers un ailleurs. Le spectacle Sans objet de Bory, mis en lumière par les outils conceptuels propres aux créations contemporaines unissant marionnette et danse, semble alors proposer un espace de résonnance entre corps de chair et corps de matière.

\section{Le choc des corps : un pas vers l'Autre}

Avec Sans objet, on assiste à une confrontation proche du science-fictionnel et du chorégraphique entre les corps de deux danseurs et celui d'un robot d'usine, homme et machine, chair et métal. Le robot, par son poids et la menace qu'il constitue, crée une tension avec la fragilité du corps de chair qui partage la scène avec lui. Les deux corporalités, vivante et non vivante, semblent se rapprocher des pratiques chorégraphico-marionnettiques contemporaines, utilisant l'objet comme corps-frontière (Beauchamp et al 10), lieu de rencontre des corps, pivot central de la représentation.

Avec ce bras d'acier qui a la capacité de se déployer pour se dresser à la verticale, le spectateur a la possibilité de projeter des fantasmes anthropomorphiques qui sont soulignés par le positionnement de deux lumières tout en haut, figurant un regard, dont la tête se plie vers l'avant et scrute la salle, faisant explicitement écho à des références de science-fiction issues du cinéma ou de la littérature : le robot, après avoir rangé, voire éliminé le corps des deux danseurs sous les planches de la scène, balaye d'un regard le public avec l'air de chercher le prochain corps humain à camoufler, déclenchant, par ce geste mi-menaçant, mi-amusant, les rires nerveux de la salle. Un contact s'établit à cet endroit entre le public et le robot, mettant en évidence, comme un sursaut de réel, le pacte implicite entre le public et tout spectacle de marionnettes, cet accord tacite attestant, non pas de la crédulité du spectateur face à la représentation théâtrale, mais de sa volonté d'adhésion à l'illusion qui lui est proposée par l'acte d'animation. La machine robotique est alors dépossédée dans son geste mécanique et utilitaire pour être ici marionnettisée, à la fois au sens d'animation - l'âme - et de manipulation, le geste technique qui crée un sentiment de vie : nous avons l'impression qu'elle regarde le public, respire, a des réactions spontanées par un moment tantôt nerveux tantôt vif et enthousiaste. 


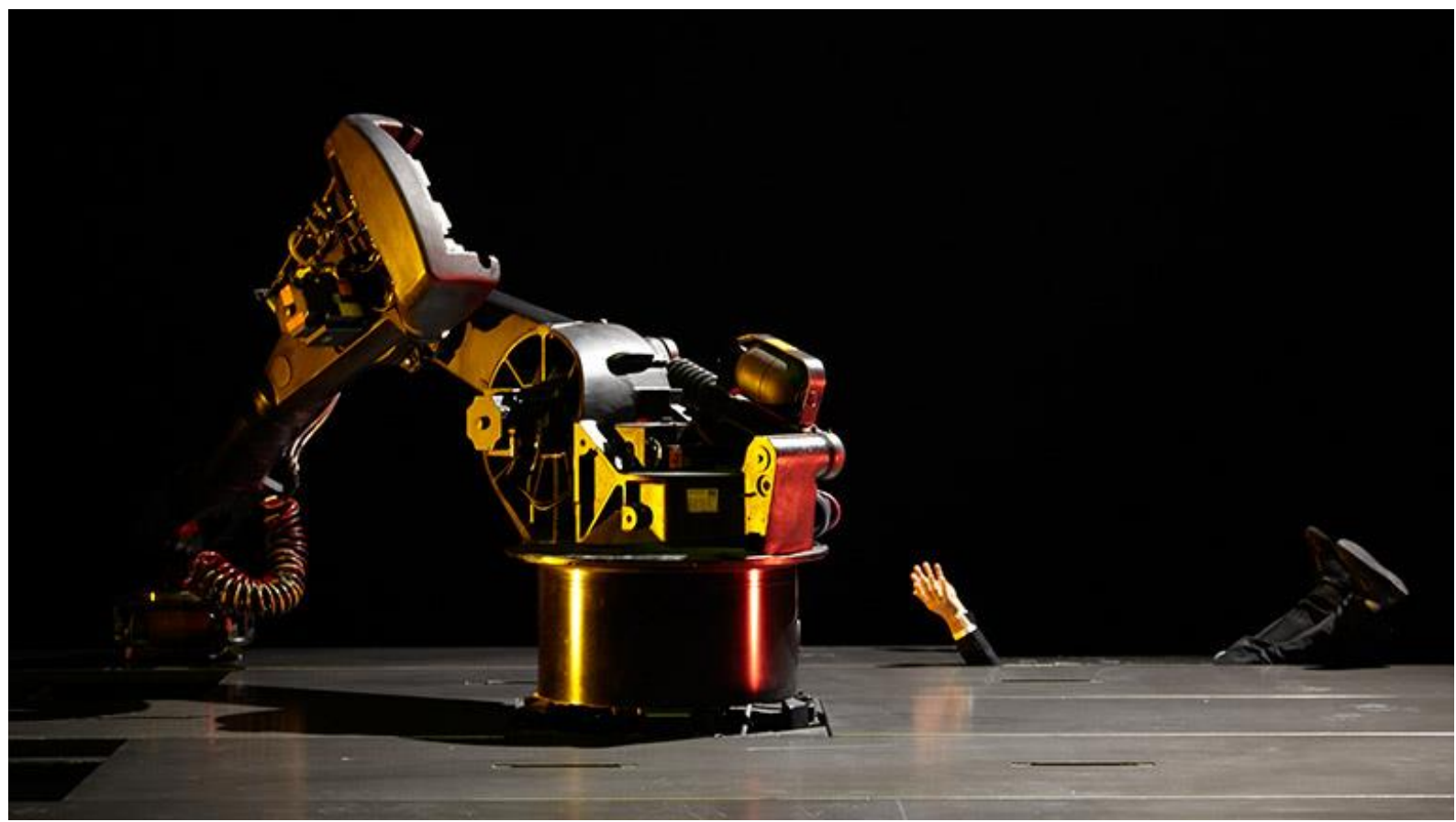

Crédits : Aglaé Bory (C)

Si la tête du robot est équipée de mors (avec des terminaisons pneumatiques dont on entend l'air comprimé s'échapper) qui s'ouvrent et permettent de déplacer les éléments du décor, il y a néanmoins un travail sur les bruitages qui vient renforcer la présence du robot, donnant à entendre le dedans de la machine, les rouages, son souffle, sa voix même s'il n'y a pas de mots, rendant le robot d'autant plus vivant. À l'inverse, les êtres a priori doués de parole que sont les danseurs, restent muets.

Les jeux sonores et lumineux permettent de voir le robot, non plus comme un agrès ou un support technique nécessaire aux danseurs, mais plutôt comme un partenaire de la chorégraphie. En reprenant la définition posée par Josette Féral (2012), puis Renée Bourassa et Louise Poissant (2013) sur la présence - qui serait alors, pour la marionnette, la manifestation du vivant sur le plateau (danseur, acteur...) interagissant avec l'objet -, le robot se positionne en effet de présence comme la marionnette, le théâtre d'objets ou le théâtre de matières, constituant un espace de projection d'imitation du vivant. En créant une illusion de vie, l'objet devient une sensation de présence, révélant ainsi trois partenaires sur le plateau (deux danseurs et un robot). Par ce procédé interactif, le spectacle bascule dans un processus oscillant entre présences et effets de présence ; dès lors, le robot devient un personnage narratif entrant en dialogue physique avec les danseurs. Ce trio chorégraphique cherche à créer un discours corporel réciproque, établissant un lien relationnel entre l'homme et la machine. Le robot devient personnage au sein de cette narrativité des corps, mais également écran de 
projection des fantasmes du spectateur qui lui imagine une tête, un regard, éprouvant de l'empathie à son égard.

Marionnette autant que marionnettiste, le robot est autant support technique nécessaire à la chorégraphie des danseurs - au sens de l'agrès propre au cirque - l'objet non vivant placé au centre de la scène, que l'instigateur du mouvement chorégraphique. Le robot apparaît dès lors comme la dynamique, la source même du mouvement. Lors d'un entretien effectué le 12 mai 2017, Bory indique à propos des répétitions de Sans objet que «Les acteurs ont été cantonnés à un rôle d'observateur pendant les trois premières semaines. Quand ils sont passés sur le plateau, je leur ai donné la consigne de suivre la machine ». En demandant ainsi à ses danseurs de n'avoir aucun contrôle sur la machine et de se laisser guider par elle, Bory place le robot comme lieu de naissance du geste chorégraphique. Le dialogue physique entre ces trois corps redistribue de manière fondamentale les relations de dominant/dominé propres aux rapports originellement établis pour la marionnette. Reprenant l'essai «Sur le théâtre de marionnettes » d'Heinrich von Kleist (1810) où la marionnette est présentée comme modèle inspirant le danseur, Bory envisage le robot d'usine comme un corps doué d'actions et de mouvements, qui parle ici le même langage corporel que les deux danseurs.

Lorsque les danseurs prennent appui sur le robot, les jeux d'ombres créent des contrastes fusionnant visuellement les trois corps en une seule et même corporalité, porteuse d'un geste lent et décomposé unique : les contours de cette silhouette deviennent hors normes, hors codes, se détachant de toute figure corporelle clairement identifiable pour créer un nouvel état de corps par un geste qui lui est propre. Par la fusion des corps qu'il propose, le contrejour des éclairages lumineux donne alors une direction à ce corps inédit, lui conférant un mouvement et un langage nouveaux.

À travers cette notion de corps-frontière mettant en avant une confrontation des corps autant qu'une fusion, il faut entendre la révélation d'une triangulaire manipulatoire allant de l'acteur à la marionnette en passant par le marionnettiste dans un mouvement spiral difficilement saisissable pour l'œil du spectateur. Nommé «machiniste» ou plus souvent « pilote » (Entretien 12 mai 2017) par Bory, un membre de la compagnie s'est formé auprès du constructeur du robot pour apprendre ses fonctions et voir de quelle(s) façon(s) cet objet industriel pouvait faire théâtre. La démarche propre à la genèse du spectacle Sans objet a donc été que les artistes sont allés à la rencontre de l'industrie pour tenter d'en adopter les codes essentiels à la création. Ce robot fait partie des plus grands de sa catégorie pouvant entrer dans une salle de théâtre : ses dimensions et sa puissance permettent l'ouverture d'une résonnance importante dans la recherche de confrontation de ces corps a priori opposés. 
Côté jardin en fond de scène, le pilote a un rôle fondamental dans la conception du spectacle, ayant révélé les possibilités et impossibilités de la machine ainsi que les risques pour parvenir à faire théâtre. Ni caché ni montré, sur le principe de la régie vue à l'extérieur du plateau de jeu tout en étant sur la scène, le visage du pilote est invisible à l'œil du spectateur, derrière l'écran de contrôle sur lequel il a un retour caméra. Sans être un personnage du spectacle, tout en ne cherchant pas sa disparition au profit de l'illusion, le pilote du robot, par son geste de manipulation assumé, est alors positionné dans une coprésence visuelle entre ces corps contraires.

Les gestes du robot sont en partie préprogrammés - à la manière d'un automate - et le pilote a la possibilité de réguler leur vitesse (par accélération ou décélération), s'inscrivant dans une temporalité très proche de celle des danseurs. Comme un marionnettiste, il donne manuellement les tops nécessaires au déclenchement de l'action. Il semble y avoir ici un double rapport à la marionnette dans la mesure où nous avons d'un côté le robot, qui est pour Bory « une marionnette technologique » (Entretien 27 avril 2017), et de l'autre les danseurs que la machine manipule, créant un rapport «d'actifs-passifs » inversé où «le robot est actif et les acteurs, les corps, sont passifs » selon l'expression de Bory puisée du même entretien.

L'humain, qu'il soit pilote ou danseur, ainsi que l'intrusion de certains éléments tels qu'une bâche (que nous évoquons plus loin) réinjectent alors dans la programmation une phase de spontanéité, dont le régulateur du temps demeure le meilleur allié : permettant de créer de l'accidentel au milieu de la programmation, le danseur et le machiniste utilisent la régularité du programme pour ouvrir vers d'autres champs d'explorations chorégraphiques.

Ce mélange entre le programme et l'accident donnait quelque chose de vivant. Le vivant fonctionne comme ça. Il y a un programme - l'ADN - et il y a l'accident : comment les conditions extérieures, comment les événements ont modifié le vivant. La façon dont le vent a plié les arbres, ce n'est pas dans l'ADN. Le vivant est toujours une combinaison d'un programme et d'un accident. (Entretien 27 avril 2017)

\section{Bouleverser le chorégraphique, tirer les corps vers la grâce}

Bory précise l'importance de la contrainte dans son travail :

Ce que signifie « suivre la machine » : comme on suit parce qu'on est contraint de suivre, parce qu'on n'a d'autre choix que de suivre les évolutions de la technologie aujourd'hui. Très peu de gens résistent, car le rapport à la technologie est que l'on n'a pas d'alternative, on doit suivre. C'est ça que je voulais comme rapport entre eux, quand les acteurs sont passés sur le plateau avec la machine : je voulais qu'ils la suivent, en leur disant « suivez-la, faites ce qu'elle vous dit de faire ». La machine a été elle-même marionnette 
technologique, mais elle est aussi marionnettiste : c'est la machine qui manipule objets et corps. (Entretien 12 mai 2017)

Les danseurs sont dans un état de corps entre vigilance et adaptation, chaque pas est investi de la conscience du geste et de son positionnement sur le robot, avec une extrême précision propre à celle des circassiens. Dès lors, la contrainte dans le rapport qui est établi ici devient source créative, permettant des explorations inimaginables sans elle.

Le robot, élément central de la chorégraphie autant que de la scénographie, constitue en réalité le centre de gravité des danseurs qui s'accrochent à lui, se suspendent ou y prennent appui, dans une relation à la fois d'humain à objet, mais aussi de partenaires chorégraphiques. En cela, le robot parvient à décaler, voire à dérouter profondément les danseurs dans leurs habitudes chorégraphiques et dans les chemins intérieurs qu'ils ont l'habitude de prendre pour créer le geste. À cet endroit, le rapport robot-humain, dans son traitement en lien avec le chorégraphique, est proprement marionnettique : on retrouve aisément ce décalage du centre de gravité, cette ouverture des potentiels créatifs chorégraphiques chez des artistes aux esthétiques différentes, mais aux processus similaires comme Ilka Schönbein - pour ne citer qu'elle - dont l'usage de prothèses marionnettiques vient prolonger et étendre son corps de marionnettiste-danseuse.

Cette extension des potentiels chorégraphiques du corps n'est pas sans faire écho à la notion de kinesphère développée par Rudolf von Laban, à propos duquel Julie Perrin (2006) dit ce qui suit :

Laban (1879-1958) est [...] le chorégraphe [...] qui a le plus clairement [...] exposé une conception cohérente de la spatialité corporelle. [...] C'était d'emblée renverser le point de vue classique sur l'espace de la danse, en faisant du corps même le référent principal à l'organisation des espaces. Un point de vue extérieur ne vient plus organiser le geste. [...] Laban insiste sur la motivation intérieure du geste et propose de réfléchir en termes de «perspective corporelle ». Cela conduit à dégager l'idée de kinesphère - cette sphère formée par tous les mouvements potentiels d'un danseur, sans transfert du corps. Délaissant tout souci d'orientation, Laban insiste davantage sur la direction du mouvement et opère un découpage analytique de l'espace en trois dimensions (verticale, horizontale et latérale), et six directions fondamentales (haut, bas, gauche, droite, avant, arrière). Cette décomposition de l'espace en vingt-six directions élémentaires donne lieu à la figure géométrique de l'icosaèdre. (5)

Dans Sans objet, il semble y avoir une extension du principe de kinesphère de Laban à la marionnette : l'objet devient un prolongement du corps du danseur partenaire, permettant d'agrandir l'étendue de sa sphère kinesphérique, ouvrant un champ de possibles explorations chorégraphiques. Le danseur parvient à s'extraire de son propre corps, pour venir imprimer son geste autrement dans l'espace. Les corps, d'acier et de chair, offrent une nouvelle graphie au 
geste chorégraphique, créant un corps commun hybride proche du science-fictionnel dont le mouvement en continu crée une métamorphose quasi infinie. On assiste dès lors à une extension du geste chorégraphique par le biais du robot, socle immuable ouvrant des possibilités aux danseurs allant jusqu'aux acrobaties : c'est alors une poétique interrelationnelle et chorégraphique qui se déploie où l'agrès devient partenaire et non plus uniquement lieu et support des prouesses spectaculaires.

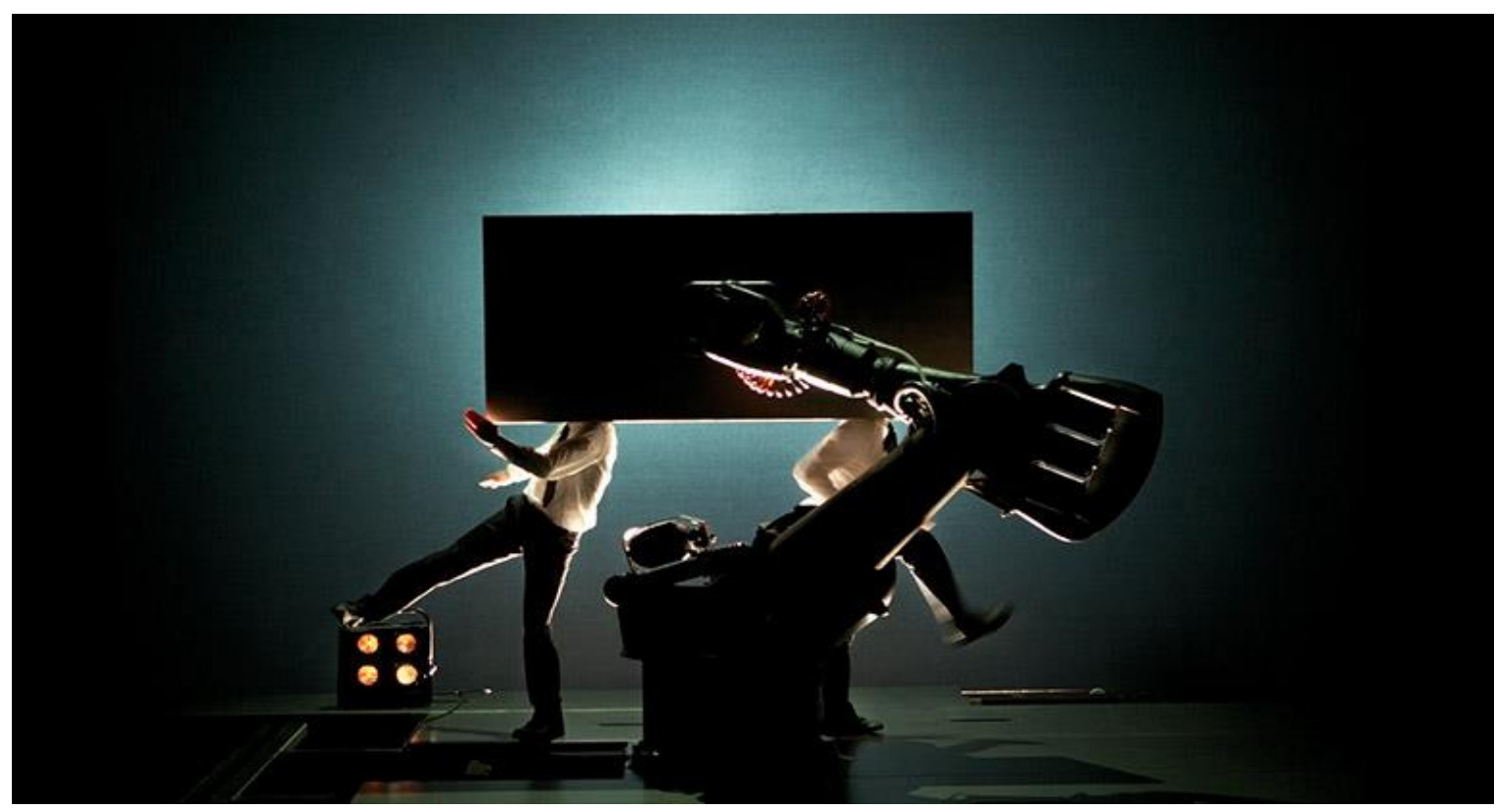

Crédits : Aglaé Bory ()

Dans l'ouvrage «Sur le théâtre de marionnettes » de Kleist, le danseur classique considère le geste de la marionnette à fils comme porteur d'une grâce incomparable détachée des lois de la pesanteur et de la gravité qui ont d'ordinaire lieu et action sur le corps vivant. Dans ce texte philosophique, la marionnette exprime ce que l'on pourrait nommer un geste de pure chorégraphie, détachant le corps de ce qui le raccroche à sa condition d'humain, ces instants qui ne sont que des manifestations des stigmates biologiques du corps et qui, selon Kleist, ne sont plus rattachés à l'idée de la danse (un corps qui retombe après un saut, les moments de repos, la reprise du souffle...) (15). Sans objet fait du robot l'instigateur du mouvement, régulateur des corps qu'il porte, éloignant l'humain (danseur ou pilote) de son rôle, à l'origine, dominant de l'action.

Bory propose avec Sans objet une approche du texte de Kleist creusant la question du rapport à la grâce. Kleist tente d'expliquer la mécanique de la grâce en faisant l'hypothèse de l'abandon, la marionnette n'ayant pas de volonté propre pour conduire son mouvement, ce qui doit devenir source d'inspiration chez le danseur. Cette grâce chez Bory se manifeste dans Sans objet avec le robot par la création d'un geste parfait. Cette perfection du geste de l'objet 
mécanique se manifeste ainsi par la réalisation technique d'un geste par exemple droit en continu sur une vitesse inchangée. Dès lors, la puissance de la machine, par son extrême précision, parvient à recréer cet idéal de geste pur évoqué par Kleist. Le geste, dans son essence première, est saisi et visuellement identifié, détaché de tout acte corporel du danseur, ou de manipulation de la part du pilote. La marque de l'humain est dès lors gommée pour atteindre l'état de grâce du geste chorégraphique.

En poursuivant cette réflexion sur la pensée théorique de Kleist, il est important de souligner que certains passages de Sans objet sont proches d'acrobaties propres au cirque. Défiant les lois de la gravité, dans des mouvements de rotation et de retournement d'un ralenti proche de l'immobilisme, le robot porte les deux danseurs avec une extrême fluidité. Les danseurs se retrouvent suspendus par un bras, les pieds vers le ciel, ou la tête en bas sans donner l'impression de fournir le moindre effort physique. Les lois de la gravité semblent bouleversées sinon anéanties dans le rapport de ce corps d'acier avec ces corps de chair, approfondissant la relation objet-humain présente initialement entre l'agrès et l'acrobate. L'humain et le robot ne forment qu'un seul corps où tout rapport de force est absent. La puissance du robot d'usine de plusieurs tonnes, au centre de la scène (localisation de l'équilibre des forces par excellence !), déjoue les lois de la gravité. Le robot devient lui-même la loi physique, remplaçant les lois universelles pour prendre une place inédite, créant une influence nouvelle sur les corps humains qu'il porte sur lui et avec lesquels, dans ce mouvement dans les airs, il ne semble former qu'une seule et même corporalité.

Dans la lecture que Bory propose du texte de Kleist, la mécanique de la grâce entend suggérer la nécessité de l'abandon de toute volonté humaine, que nous pourrions qualifier d'un certain déterminisme chez l'individu (prédéterminant a priori nos actions par l'enchaînement de la cause et de l'effet). Bory précise que : «La marionnette [chez Kleist] nous apprend quelque chose au-delà de la volonté humaine » (Entretien 27 avril 2017). Les corps humains choisissent ici de se livrer entièrement à la machine. Un choix non sans conséquence : les préparations du spectacle ont été plus que fastidieuses dans la mesure où il a fallu apprendre à dompter cette machine, programmer ses mouvements, les attester d'un point de vue strictement sécuritaire avant de pouvoir introduire des corps dessus. Ce n'est qu'après ce long processus que les danseurs ont pu trouver un espace d'improvisation et de création, pour parvenir à faire corps avec le robot. 


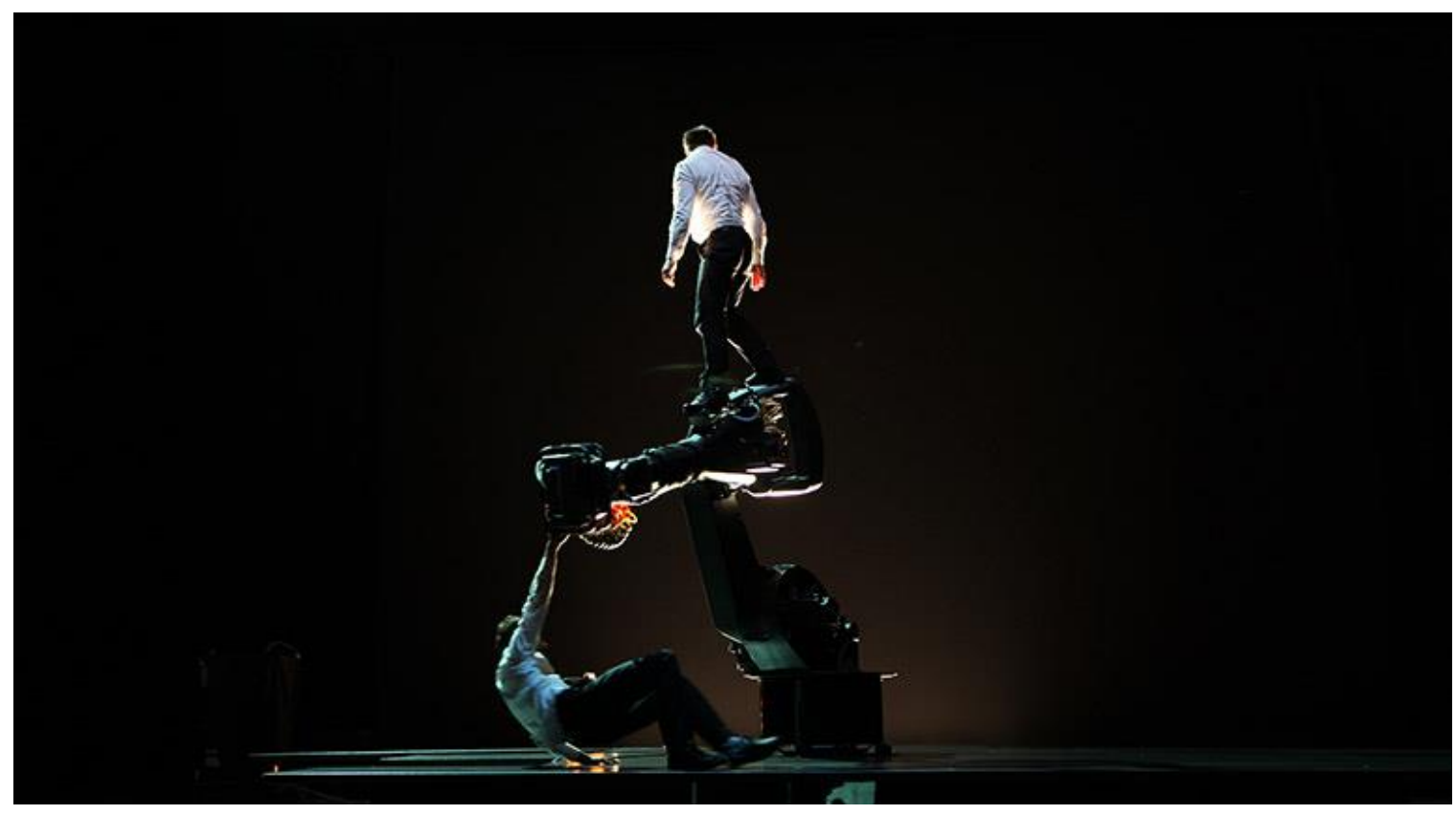

Crédits : Aglaé Bory @

\section{Quand le geste répond à l'espace}

Dans Sans objet, il y a en réalité une scène sur la scène. Très concrètement, il a fallu que l'équipe s'interroge sur la façon de faire tenir ce robot d'acier sur une scène de théâtre dont les planchers ne supportent que trois cent cinquante kilogrammes au mètre carré. Dans l'industrie, ces machines sont fixées sur des sols de béton par une vingtaine de vis d'un mètre de long chacune. La scénographie du spectacle est donc née de cette difficulté technique, amenant à la création d'un socle propre au robot répartissant la charge sur toute la surface, plaçant le robot en suspension totale par rapport au plancher de théâtre. La statue (par dérivé de la marionnette) que constitue alors le robot dans Sans objet, trouve ici son socle, nécessaire à son exposition, faisant écho aux sculptures de bronze, notamment celles d'Auguste Rodin sur la danse (inventant des postures déséquilibrées issues du fruit de son imagination qui, par la suite, ont été expérimentées par des danseurs professionnels) ${ }^{3}$ ou bien encore à La valse (sculptée entre 1889 et 1905) de Camille Claudel où les corps des deux danseurs sont suspendus dans une posture de déséquilibre improbable, véritable prouesse technique chorégraphique autant que sculpturale.

Par ce socle, l'espace devient modulable dans la mesure où chaque planche qui le constitue, peut se retirer. Le robot, par les mors qui ont été évoqués précédemment, insuffle un mouvement nouveau à l'espace et ouvre des possibilités aux danseurs. Il transforme l'espace à sa guise, offrant des cachettes, des trappes, des plateaux élévateurs ou des panneaux de camouflage aux danseurs. Comme souvent chez Bory, l'espace est marionnettisé, devenant matière vivante en perpétuelle métamorphose mouvante. Dans la précédente création de 
l'artiste intitulée Questcequetudeviens (2008), on assistait déjà à des changements d'état de l'espace par l'eau: tantôt liquide au sol, tantôt vapeur dans un préfabriqué vitré, tantôt bouillonnante lorsqu'elle est piétinée, les étapes successives de l'eau s'enchaînent lors de son contact avec le corps de la danseuse Stéphanie Fuster, rendant l'espace vivant et évolutif, une constante que l'on observe chez Bory.

Au début du spectacle, tel un prologue, intervient un autre objet, le seul présent sur l'ensemble de la représentation en dehors du robot, que l'on retrouve également à la fin. Il s'agit d'une bâche agricole destinée, à l'origine, à recouvrir les champs. Alors que le public vient de s'installer, la scène est animée par une masse noire, informe, aux bruits métalliques. Pendant plusieurs minutes, cette masse de bâche, comme animée par un feu interne, tourne, se baisse, se plie, se tend, se froisse. Une chorégraphie de l'intérieur semble bouillir et vouloir éclater. Happé par cet effet visuel, l'œil du spectateur entre quasiment dans un état d'hypnose, projetant autant d'images, autant de visages, sur les plis imparfaits et aléatoires de cette bâche. Cet effet hypnotique - constaté lorsque le dispositif est présenté lors d'installations (pensons notamment à l'exposition Prosopopées au Centquatre à Paris en 2015 dans laquelle le robot, sans machiniste, était programmé) -, par les extraordinaires qualités de projections qu'il propose, fabrique une nouvelle matière de corps : ni matériau, ni vivant, ni non-vivant.

Dans cette masse informe de la bâche, dont le mouvement en perpétuelle mutation est provoqué par le robot présent en dessous, viennent se rencontrer la perfection du geste du robot et les plis imparfaits de la bâche plastique que Bory qualifie d' « accidentels » (Entretien 12 mai 2017). C'est ce travail de l'accident face à la programmation qui fut ensuite testé sur les corps des danseurs dans leur rapport au robot pour élaborer la chorégraphie de Sans objet. D'un élément a priori scénographique et autonome (ne nécessitant qu'une programmation) a pu naître le geste chorégraphique propre aux vivants du spectacle, créant ce lien interactif où le robot se place en pivot central, en médiateur.

Cette bâche qui englobe le robot n'est pas sans faire écho de nouveau aux bronzes de Camille Claudel et, en particulier, à la barbe du Buste d'Auguste Rodin (1892), travaillée à la fois comme le lieu de l'inachevé et le lieu de l'émergence des formes, entre brutalité de la matière et matériau volontairement laissé comme évanescent. De la même façon, le chanvre utilisé comme matière première, lieu de naissance des formes et des corps dans L'orée des visages (2013) des compagnies Pseudonymo et CFB451, le bronze de Claudel qui, d'un point de vue purement technique, vient après la phase de sculpture, résonnent ici avec le plastique de Sans objet qui cache le robot. «Ce magma originel, cette forme d'avant la forme » (Entretien 
12 mai 2017) vient alors, comme un matériau premier, révéler la forme finale que constitue le robot en dessous, faisant résonner la mécanique interne.

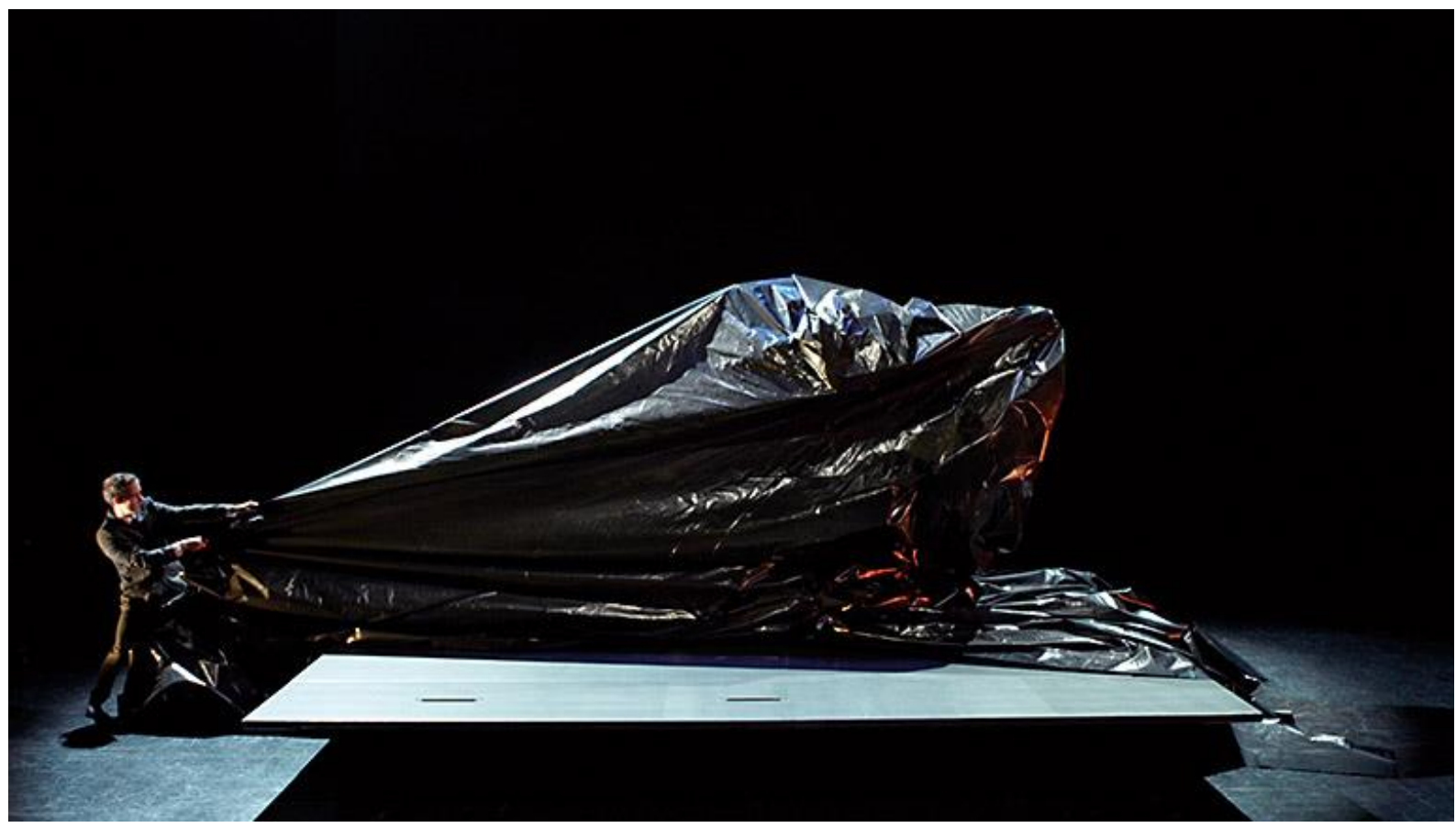

Crédits : Aglaé Bory @

Alors, il devient désormais possible d'envisager la chorégraphie comme une réponse à la scénographie créant une autre matière de corps. Chez Bory, en fondant la dramaturgie (ceci de manière générale dans ses spectacles) sur un processus de transformation, la métamorphose devient le maître mot de Sans objet. Plongé dans l'incertitude la plus totale, dans la semi-obscurité, baigné de sonorités étranges aux sources inconnues, le spectacle Sans objet vient combler les fantasmes science-fictionnels du spectateur, allant vers un au-delà des clichés littéraires et cinématographiques pour apporter des corps non plus contraints ou situés dans un rapport de force, mais bien des rencontres d'effigies différentes dans un espace instable.

Ce processus de transformation amène, de fait, à la question du déplacement, pensé à la fois comme un déplacement physique - le robot décontextualisé de son usine - et un déplacement du regard - celui que l'humain (danseur ou spectateur) porte sur ce robot une fois placé au centre de la scène, devenant objet théâtral -, qui fait ressortir nécessairement les corps de la représentation profondément modifiés, métamorphosés dans leur essence. Il s'agit toujours pour Bory de mettre les choses au bord, au seuil, voire à la frontière (ce qui rejoint cette question du corps-frontière que nous avions développée au début) : cette zone instable de l'ailleurs encore inexploré devient pour Bory le lieu du questionnement, le lieu de la création.

Avec Sans objet, Bory opère la rencontre fusionnelle du robot d'usine et de l'homme. Cette confrontation des corps, entre vivant et non-vivant, chair et acier, crée une coprésence 
chorégraphique où naît une sensation de geste pur. Les danseurs, dans un acte d'abandon, suivent sans retenue le geste parfait et mécanique du robot, créant un geste commun où seuls les corps dominent. L'esprit (qu'il soit celui des danseurs ou du pilote) semble avoir quitté les corps pour venir atteindre l'état de grâce tant cherché par Kleist.

En apprenant le langage de l'Autre, les danseurs parviennent à humaniser le robot, dont les projections anthropomorphes de la part du spectateur sont fréquentes quoique parcellaires. Le poids et la densité des trois corps disparaissent pour plonger dans une fluidité insaisissable et incertaine, faisant naître et mourir des images aussi fugaces les unes que les autres, à la manière des marionnettistes utilisant du matériau brut pour faire émerger des éclats de vie aussitôt évaporés : «L'essentiel n'est plus le respect des codes établis mais la nécessité d'une expression émise ici et maintenant. [...] Le corps glorieux est défunt, son double aussi. Danse ou marionnette, ce sont des prétextes à une déstabilisation excitante qui permet aux artistes d'être là où on ne les attend pas » (Lecucq 3 ).

\section{Bibliographie}

Alvarez, Gilles et José-Manuel Gonçalvès, dir. Prosopopées, quand les objets prennent vie. Exposition dans le cadre de la 15e Biennale internationale des arts numériques (NÉMO), Le Centquatre, Paris, 5 décembre 2015-31 janvier 2016.

Beauchamp, Hélène, Joëlle Noguès et Élise Van Haesebroeck, dir. Marionnette, corps frontière. Mayenne : P Artois U, 2016.

Bory, Aurélien. Sans objet. Dossier de presse, 2009.

Bourassa, Renée et Louise Poissant, dir. Avatars, personnages et acteurs virtuels. Québec : PU du Québec, coll. Esthétique, 2013.

Chevillot, Catherine (Commissaire). Rodin. L'exposition du centenaire. Exposition, Grand Palais, Paris, 22 mars-31 juillet 2017.

Féral, Josette, dir. Pratiques performatives, body remix. Rennes : PU de Rennes et PU du Québec, 2012.

Kleist (von), Heinrich. «Sur le théâtre de marionnettes ». Tr. de l'allemand Jacques Outin. Paris : Mille et une nuits, 1993, [1810].

Lecucq, Evelyne. «Flagrant délit de décalage ». Objet-Danse. Dir. Evelyne Lecucq et Sylvie Martin-Lahmani. Bruxelles : Alternatives Théâtrales, $2003: 3$.

Martin, Nicolas et Aurélien Bory. «Pas de danse avec Aurélien Bory ». Entretien réalisé par France Culture. 15 juillet 2015. https://www.franceculture.fr/emissions/l-inviteculture/pas-de-danse-avec-aurelien-bory. Consulté le 30 novembre 2017. 
Pavis, Patrice. «Médialité et intermédialité ». Dictionnaire de la performance et du théâtre contemporain. Paris : Armand Colin, 2014 : 151-153.

Perrin, Julie. «L'espace en question ». Repères, Cahier de la danse 18 (novembre 2006) : 3-6.

Peydro, Laurence. «L'univers blanc des plâtres de Rodin ». Rodin. L'exposition du centenaire.

Anne de Séror (Secrétaire de rédaction). Paris : «Beaux-arts » hors-série, (2017) : 3441.

Plassard, Didier. «Aurélia Ivan. Interroger le mouvement ». ArtPress 238 (août 2015) : 6466.

\section{Chorégraphies}

Boivin, Dominique. Transports exceptionnels, duo pour un danseur et une pelleteuse. 2005.

Bory, Aurélien. Questcequetudeviens. Bordeaux, novembre 2008.

---. Sans objet. Toulouse, 2009.

Girondin-Moab, David, Angélique Friant, Christian et François Ben Aïm. L’orée des visages. Charleville-Mézières, septembre 2013.

\section{Entretiens inédits}

Bory, Aurélien. Entretien inédit avec Oriane Maubert. 27 avril 2017.

---. Entretien inédit avec Oriane Maubert. 12 mai 2017.

\footnotetext{
Notes

${ }^{1}$ Bory se forme d'abord aux sciences physiques à l'Université de Strasbourg, ce qui le conduit premièrement à travailler sur l'acoustique architecturale. En 1995, il intègre le studio de création au sein du Lido, Centre des arts du cirque, à Toulouse. C'est en 2000 qu'il fonde la Compagnie 111 dont les créations s'orientent vers un théâtre curieux, physique, très singulier, à la croisée des arts et des genres, échappant, du coup, à toute forme d'identification disciplinaire. La Compagnie 111 touche ainsi autant au cirque qu'à la danse, aux arts visuels, etc. ${ }^{2}$ Dans ce présent travail, élément d'une thèse de doctorat préparée à 1'Université Montpellier 3 Paul-Valéry et intitulée «La marionnette danse : réactiver le geste sur la scène occidentale contemporaine », le robot tient une place à part, appartenant à la fois au large champ des arts de la marionnette, et se situant quelque peu à la marge. Ce qui pourrait différencier le robot de la marionnette est sans doute, outre sa confection et l'imaginaire qui en découle, la programmation technologique qu'il implique nécessairement. Il rejoint néanmoins, dans le présent article, la marionnette par son usage spectaculaire d'abord, la part de spontanéité et d'improvisation laissée dans la programmation par la manipulation en direct ensuite, et enfin par la rencontre dialoguée, cet effet retour entre l'interprète et l'objet, qui constitue l'un des éléments clés de la marionnette au théâtre. Un outil donc que l'on manipule tout en le programmant, qui soutient en tant qu'objet autant qu'il peut donner du jeu, donner le sentiment de surprendre, presque comme un partenaire.

3 À cet égard, voir les ouvrages de Catherine Chevillot (2017) et de Laurence Peydro (2017).
} 